\title{
Application of 2-D inversion with genetic algorithms to magnetotelluric data from geothermal areas
}

\author{
Marco A. Pérez-Flores ${ }^{1}$ and Adam Schultz ${ }^{2}$ \\ ${ }^{1}$ CICESE/Earth Sciences, Km 107 Tijuana-Ensenada, Ensenada 22860, B.C., México \\ ${ }^{2}$ Cardiff University, Department of Earth Sciences, Cardiff, CF10 3YE, United Kingdom
}

(Received October 23, 2000; Revised January 31, 2002; Accepted February 21, 2002)

\begin{abstract}
We apply a modified genetic algorithm, the "recombinant genetic analogue" (RGA) to the inversion of magnetotelluric (MT) data from two different geothermal areas, one in El Salvador and another in Japan. An accurate 2-D forward modelling algorithm suitable for very heterogeneous models forms the core of the inverse solver. The forward solution makes use of a gridding algorithm that depends on both model structure and frequency. The RGA represents model parameters as parallel sets of bit strings, and differs from conventional genetic algorithms in the ways in which the bit strings are manipulated in order to increase the probability of convergence to a global minimum objective function model. A synthetic data set was generated from a chessboard model, and the RGA was shown capable of reconstructing the model to an acceptable tolerance. The algorithm was applied to MT data from Ahuachapán geothermal area in El Salvador and compared with other interpretations. Data from the geothermal area of Minamikayabe in Japan served as a second test case. The RGA is highly adaptable and well suited to non-linear hypothesis testing as well as to inverse modelling.
\end{abstract}

\section{Introduction}

This paper reports on the application of a genetic algorithm (GA) to the nonlinear inversion of magnetotelluric data from the geothermal areas of Ahuachapán, El Salvador and Minamikayabe, Japan. Genetic algorithms were first applied to the magnetotelluric problem by Everett and Schultz (1993). In the present work, we have applied an improved forward modeling method. In the previous work the grid used to solve the governing differential equation was frequency dependent according to a simple scaling law, i.e. a denser grid was used at higher frequencies. In the work described here we used the 2-D Cartesian forward modeling algorithm developed by Weaver and co-workers (Weaver and Brewitt-Taylor, 1978; Poll, 1994). The genetic algorithm demands accurate forward solutions for models that can at times be very heterogeneous and have large conductivity contrasts. After tests with such models we found the Weaver forward modeling algorithm more stable numerically than alternatives. This stability arises in part because the grid to solve the TE and TM mode is both frequency and model dependent. Accurate solutions are found for both modes, even for high conductivity contrasts. The algorithm discretizes only the earth half-space for the TE mode by means of integral boundary conditions (Weaver, 1994), with the advantage that the forward solution is very fast.

The rapid solution and the automatic gridding features of the forward algorithm are particularly useful for GA-based inversions, because in each iteration ("generation") of the inversion a set of quasi-randomized models are proposed, with

Copy right (C) The Society of Geomagnetism and Earth, Planetary and Space Sciences (SGEPSS); The Seismological Society of Japan; The Volcanological Society of Japan; The Geodetic Society of Japan; The Japanese Society for Planetary Sciences. attributes inherited from the previous generation of models, according to a bit string encoding particular to GAs and related algorithms (e.g. Everett and Schultz, 1993). The models whose responses are closer to the observed data, subject to a number of side conditions, survive and transmit their winning characteristics to the next generation of models. The measure of closeness is determined by a norm applied to an objective function. An objection function can be selected to minimize model roughness, so that the initial collection of very heterogeneous models can converge to increasingly smoother models, with response functions increasingly closer to the data being inverted.

The genetic algorithm applied here differs from that of Everett and Schultz (1993). The new algorithm, a "recombinant genetic analogue" (RGA) uses a different model representation than the conventional GA, with model characteristics represented by parallel sets of bit strings rather than the single string used conventionally. The process of "crossover" involves splitting the bit strings along parallel strands as well as permitting individual strands to be split longitudinally, as in e.g. Everett and Schultz. We find this method improves convergence properties significantly. Heterogeneity in model attributes is also encouraged through the process of "mutation" or random parity changes in individual bit strings during the cross-over process. At later stages of the inversion, we fine-tuned the convergence properties by permitting only one mutation per model (bit string) instead of several. We also introduce a priori constraints for some of the model parameters (prism resistivities) in order to introduce independent information, e.g. from boreholes, or to represent the sea as in the coastal Minamikayabe data.

A test with synthetic data contaminated by noise shows 
us the self-consistency of the RGA. The model obtained for Ahuachapán resembles previously published models. We have also compared our results for the Minamikayabe data against a model proposed by Cerv and Pek (1997), and lithological data from two boreholes. These comparisons demonstrate that the RGA is a viable, highly flexible, albeit computationally-intensive alternative to linearized inversion methods. The algorithm is also extremely well-suited to parallelization, promising nearly linear speed improvements as a function of the number of available processors.

\section{GA/RGA Requirements for Solution of the For- ward Problem}

In contrast to many inverse methods, nonlinear inversions based on the GA/RGA method do not require explicit or implicit calculation of the Hessian matrix. There is no direct knowledge of the slope of the misfit surface, rather information about the ability of a given model to fit the data is stored implicitly, and entirely within the bit string encoding of the model's features. There is effectively "genetic" knowledge of how well a given population does or does not fit a given data set. This lack of knowledge of how previous models have mapped onto a misfit surface has the consequence that there is no direct knowledge of steepest descent paths. As a result, convergence to a model with desirable misfit features tends to be dramatically slower than for steepest descent, Newton or other related minimization algorithms. The particular genetic encoding of GAs/RGAs, and the cross-over and mutation process provides the advantage however, that GAs/RGAs are far more resistant to descending into a local minimum. They share this feature with purely stochastic Monte Carlo methods, yet have demonstrable convergence properties, i.e. in all but the most pathological test cases thus far, we find that the RGA will converge close to the global minimum in a countably finite time.

For 2-D MT inversions based on the GA/RGA, we therefore need not require the forward solution to calculate derivatives of the fields with respect to changes in model parameters. Rather we need only calculate the $\mathbf{E}$ field from the TE mode and the $\mathbf{B}$ field from the TM. From these fields we derive the complex impedances and the apparent resistivities and phases for both modes. After testing with several codes we found that Weaver and co-workers' method was the most useful for our purpose (Brewitt-Taylor and Weaver, 1976; Poll, 1994), because this makes use of an automatically generated grid that depends both on the details of the model as well as on the frequency. The grid is denser for increasingly heterogeneous models, and for higher frequencies. The study area is discretized as a set of 2-D prisms of infinite extent in the $x$-direction. The grid used to solve the governing differential equations depends within each prism on the skin depth appropriate to the resistivity of the prism (Weaver, 1994). The 2-D domain is imbedded in an automatically-generated 1-D model at either side of the study area. The domain is bounded below by a constant resistivity half-space whose resistivity is the geometric average of the adjacent prism resistivities. For the TE mode an integral boundary condition is applied in order to avoid discretizing the overlying air half-space (Weaver, 1994).

\section{Recombinant Genetic Analogue}

Genetic Algorithms are quasi-stochastic optimization techniques. GAs solve complex optimization problems by mean of proposing random hypotheses, and selecting from a population of models only those models with the most desirable features. The desirability of the features within a model is defined mathematically by applying a norm to an objective function (e.g. desirable models may be those whose apparent resistivity and phase curves are of minimum RMS misfit to a given data set, etc.). Models with undesirable features are not permitted to pass on those features to successive iterations of the inversion. The method used in GAs and in the present RGA distinguishes them from other "evolutionary" algorithms. The model parameters (resistivities, prism geometries etc.) are coded in the form of bit strings, representing in a simple way the structure of chromosomes in a genome. The optimization process itself attempts to mimic natural selection within biological populations.

The details of the conventional GA are found in Everett and Schultz (1993). As noted in the introduction, in the algorithm used here (the RGA), the single bit string "strand" used to represent the parameters within a single model has been replaced by two parallel strands. Each model parameter is encoded as two parallel bit strings ("genes") of a finite, user-selectable length. These are appended end-toend to form a complete "chromosome", or representation of all of the parameters comprising a given model. The number of bits in each parallel strand determines the fineness of resolution of the given parameter (e.g. an 8-bit long string can represent only 256 states, a 20-bit long string more than 1 million states). Excessively short strings may make it impossible to represent the parameters adequately, inhibiting convergence of the GA to a globally optimal model. Excessively long bit string representations may slow down the convergence of the algorithm unnecessarily. The mapping between the bits in the two parallel strands and the value of any given parameter (e.g. the resistivity of a particular prism) follows a user-definable function, e.g.

$$
\log _{10}\left(\rho_{j}\right)=s_{j 1} \quad \& \quad s_{j 2},
$$

where $\rho_{j}$ is the resistivity of prism $j, s_{j 1}$ is the bit string of the first parallel strand, $s_{j 2}$ is the bit string of the second parallel strand, and \& is the bit-wise Boolean operator. This mapping is an extremely crude attempt to mimic in biological terms the "expression" of genes, where genes are determined by characteristics inherited from two parent models.

During each iteration, the link between the parallel strands is broken, and one half of each strand is exchanged, or "crossed-over" with a corresponding strand of another, randomly selected model, or "partner" from the population of models. During cross-over, sections of each strand can randomly, and according to a controllable probability function, be excised and transposed within the original strand. At the same time, random parity reversals can occur within any bit within each strand, according to a controllable probability function. This "mutation" process increases the genetic diversity of the population and is seen to counteract a process of premature "quenching", or convergence of the population to an excessively heterogeneous set of sub-optimal models. 
Table 1. Similarities between natural and geophysical optimization.

\begin{tabular}{|c|c|}
\hline Biological adaptation & Geophysical inversion \\
\hline An Individual & A model \\
\hline A Chromosome represents a DNA chain. & A parameter is the resistivity of one prism. \\
\hline A Gene is a piece of a DNA chain. & $\begin{array}{l}\text { A chain of binary numbers represents every } \\
\text { parameter value. }\end{array}$ \\
\hline $\begin{array}{l}\text { As the generations pass, the individuals are } \\
\text { getting better adapted. }\end{array}$ & $\begin{array}{l}\text { As the iteration advances, the model responses fit } \\
\text { better the data. }\end{array}$ \\
\hline $\begin{array}{l}\text { A population of " } n \text { " females and " } n \text { " males is } \\
\text { selected. }\end{array}$ & $\begin{array}{l}\text { Two random groups of models are defined. Same } \\
\text { size in both }(n) \text {. }\end{array}$ \\
\hline $\begin{array}{l}\text { Random weddings are done. Every pair has a } \\
\text { child, but the number of males is equal to } \\
\text { number of females. }\end{array}$ & $\begin{array}{l}2 * n \text { pairs of models are selected randomly and } \\
\text { mixed to get just one from every pair. } 2 * n \text { models } \\
\text { are obtained and divided in two groups. }\end{array}$ \\
\hline $\begin{array}{l}2 * \mathrm{n} \text { random pairs of individuals are selected } \\
\text { and the best adapted from every pair is selected } \\
\text { to continue living. From these survivors, the } \\
\text { global best and the global worst are found. A } \\
\text { duplicate of the global best is placed where the } \\
\text { worst is. }\end{array}$ & $\begin{array}{l}2 * n \text { random pairs of models are chosen and those } \\
\text { with the least misfit are kept. From the remaining } \\
\text { models the ones with the lowest and largest misfit } \\
\text { are found. A duplicate of the lowest misfit model } \\
\text { is placed where the largest is. }\end{array}$ \\
\hline $\begin{array}{l}\text { From the } 2 * n \text { survivors, two groups are organized. } \\
\text { The group of males and the group of females. }\end{array}$ & $\begin{array}{l}\text { The resulting } 2 * n \text { models are grouped randomly } \\
\text { in two sets. }\end{array}$ \\
\hline $\begin{array}{l}2 * n \text { random weddings are organized between } \\
\text { the two groups. More than one marriage is } \\
\text { allowed in order to assure } 2 * n \text { descendants. }\end{array}$ & $\begin{array}{l}\text { Random pairs between the two groups are obtained } \\
(2 * n) \text {. From every pair, a single model is derived. }\end{array}$ \\
\hline $\begin{array}{l}\text { After some generations the population becomes } \\
\text { homogeneous and no new characteristics appear. } \\
\text { Genetic mutation is allowed to avoid that. }\end{array}$ & $\begin{array}{l}\text { After some iterations, rapid convergence can } \\
\text { reach a local minimum. A mutation parameter is } \\
\text { allowed in order to continue searching for other } \\
\text { minimums. }\end{array}$ \\
\hline $\begin{array}{l}\text { By this process we expect that successful } \\
\text { descendants will survive and the genetic } \\
\text { information of these will assure an optimum } \\
\text { adaptation to the medium. }\end{array}$ & $\begin{array}{l}\text { We expect that in every iteration, the remaining } \\
\text { models will fit the data better, until no more } \\
\text { convergence is obtained and the searching process } \\
\text { stops. }\end{array}$ \\
\hline
\end{tabular}

While apparently ad hoc in detail, the GA and its successor the RGA are designed to mimic on the chromosomal scale the essential features of sexual reproduction ("crossover" and "mutation"), and on the population scale the process of natural selection. There has been considerable interest in the area of evolutionary algorithms, such as the Neighbourhood Algorithm (NA) (Sambridge, 2001). There is some evidence that biological mimics such as GAs and RGAs may have slower convergence properties overall than some alternatives, such as NAs. While this has not been established formally, it may transpire that biological algorithms impart an additional feature to the model search process that other approaches do not. Biological systems must optimize their organization on two separate scales, that of the individual and that of the population at large. For the case of the individual, the tendency to survive selection so as to reproduce into succeeding generations is a purely local calculation that depends on immediate factors (or in math- ematical terms, on optimizing an objective function, e.g. minimum misfit, etc.). In terms of the overall population, however, biological systems must also select for adaptability. Excessively specialized populations with insufficient genetic diversity will tend to be vulnerable to extinction in the presence of environmental change. If truly successful in mimicking the process of biological selection, it may be that GAs will tend to select for populations of models capable of future adaptation (genetic diversity) as well as capable of minimizing undesirable features. This may well tend to moderate the evolutionary process, so that in terms of geophysical inversion, the GA and RGA may tend to converge more slowly, but be more resistant to descending into local minima than alternative algorithms. Unfortunately, no schema has yet been devised to put this tendency into a more quantitative basis. In Table 1, we summarize the similarities between adaptation and geophysical inversion.

In the present work we need to solve the nonlinear inverse 


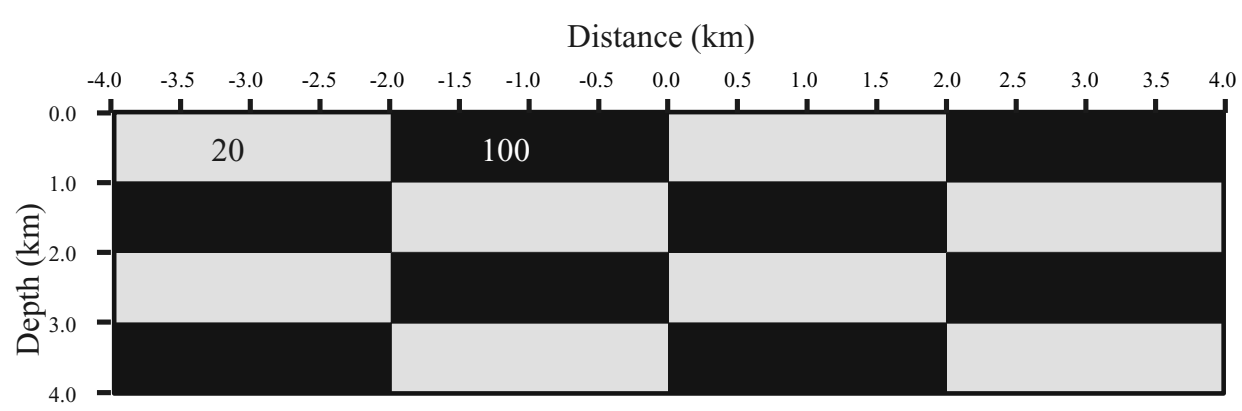

(a)

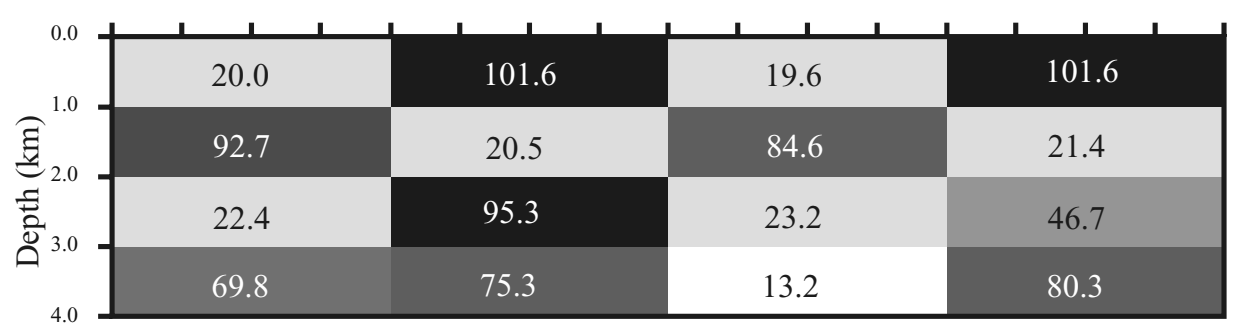

(b)

Fig. 1. (a) Synthetic model. Dark represents $100 \mathrm{ohm}-\mathrm{m}$ and clear $20 \mathrm{ohm}-\mathrm{m}$. (b) Model recovered after 330 generations. Numbers represent the estimated resistivities.

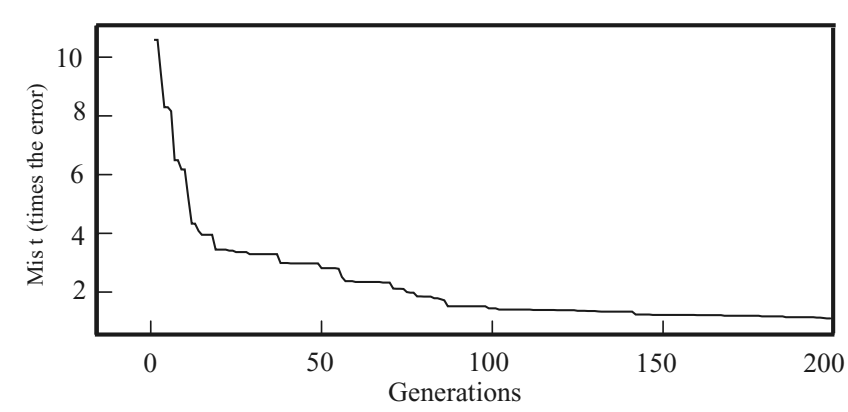

Fig. 2. Convergence plot. There is rapid convergence at the beginning and then the improvements asymptote as the population matures.

problem for magnetotelluric imaging. We assume a Cartesian Earth consisting of a set of 2-D prisms, each with variable resistivity. It is possible to encode the geometry of the prisms as part of the "genome," and to invert simultaneously for both prism resistivity and geometry. For the present work we have used fixed model geometry and have permitted only the resistivity of the prisms to be free parameters in the inversion. We have chosen to do this to reduce the number of free parameters, and to accelerate the convergence process.

The RGA seeks to best fit the observed apparent resistivity and phase data, according to rules described below, for both modes (TE and TM), at several frequencies and observation sites. The forward modeling algorithm computes the $\mathbf{E}$ and $\mathbf{B}$ fields for each frequency. The computational time scales linearly with the number of frequencies. The relationship between the number of surface observation sites, the number of free parameters and the convergence rate is more complicated, and depends strongly on the heterogeneity of the target from which the data were collected.

\subsection{The objective function}

The RGA seeks to minimize an objective function $(F)$ in order to select for models with desirable features. The objective function can be arbitrarily complicated. In the simplest case, it could comprise the norm of the misfit. Many side conditions can be introduced however. One could minimize the norm of the misfit while also minimizing the roughness of the model, and the "whiteness" of the misfit (that is, finding models that have these features while simultaneously neither overfitting nor underfitting either high or low frequency data). We have had considerable success in applying these concepts to the 1-D inverse problem, and thereby finding models with maximally smooth conductivity-depth profiles that fit the data in both the simplest, or RMS sense, and also in the sense of higher order moments such as slope of misfit vs. frequency. Prior to recent and dramatic increases in computing power, the relative computational expense of the 2-D problem made it undesirable to slow the convergence of the 2-D inversion by adding such side conditions. As a result, in the work reported here, the objective function consists simply of the quadratic norm of the difference between the observed (obs) and calculated (cal) apparent resistivity $\left(\rho_{a}\right)$ and phase $(\phi)$ for both modes (TE and TM), as shown in (2)

$$
F\left(\rho_{i}\right)=\left\|\rho_{a}^{\mathrm{obs}}-\rho_{a}^{\mathrm{cal}}\right\|^{2}+\left\|\phi^{\mathrm{obs}}-\phi^{\mathrm{cal}}\right\|^{2} .
$$

The algorithm we have used has been designed flexibly so that more elaborate objective functions, as described above, can be implemented trivially. The amplitude and phase differences are normalized by the standard error in the data. Only the $N / 2$ models within the population of size $N$, whose objective function value is within the lowest $50 \%$ are permitted to pass their characteristics on into succeeding generations. As the algorithm iterates, there is a tendency for the values of $F$ of the surviving models to decline. The 


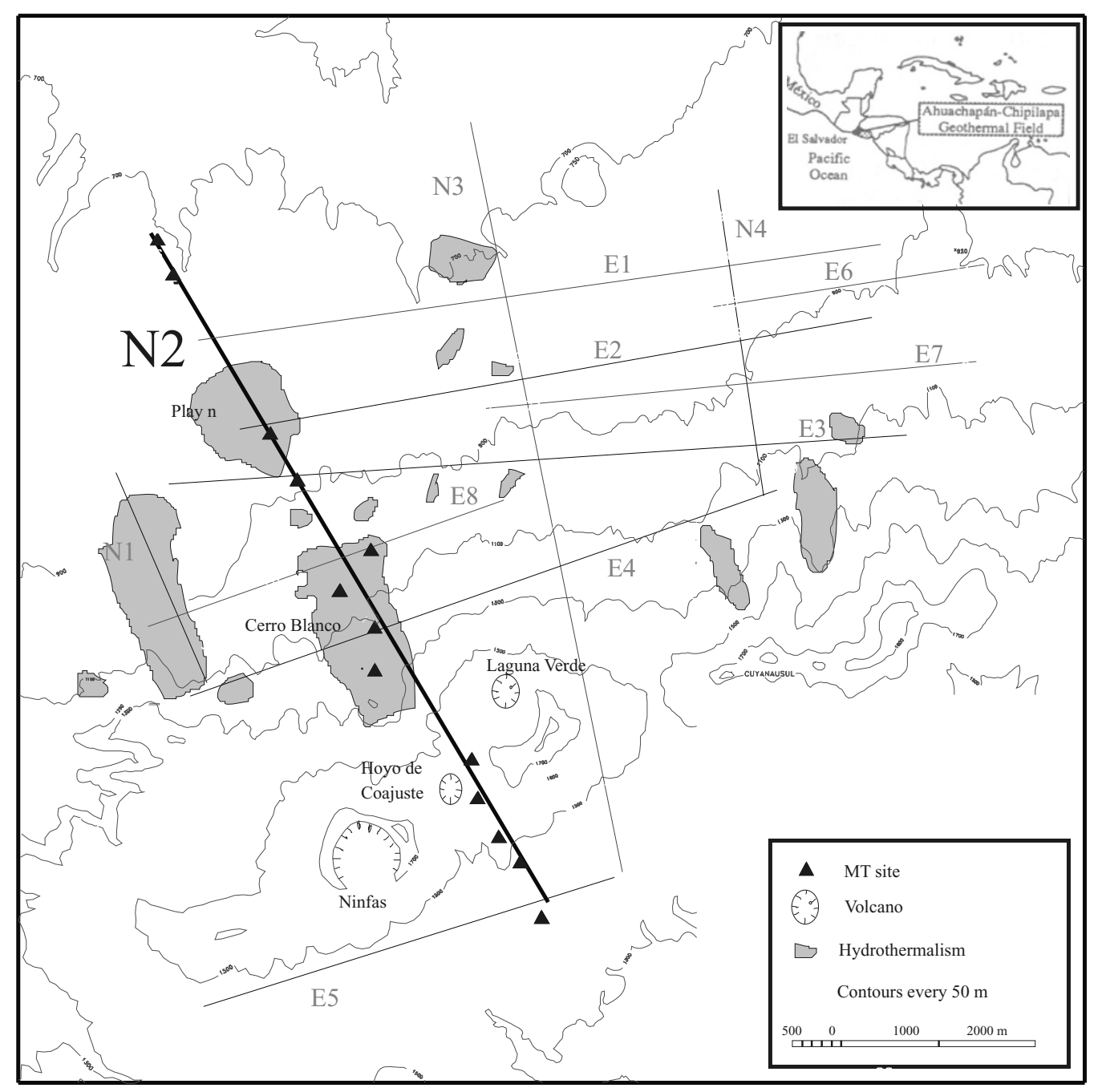

Fig. 3. Location of the Ahuachapán geothermal area, the MT soundings and the N2 line.

process continues until the following criterion is fulfilled.

$$
1.0 \leq \frac{1}{N}\left[\sum_{1}^{N} \frac{\left(Y_{\mathrm{obs}}-Y_{\mathrm{cal}}\right)^{2}}{C_{Y}^{2}}\right] \leq 1.2
$$

where $Y_{\text {obs }}$ represents the observed data, $Y_{\text {cal }}$ the response of the model and $C_{Y}$ the standard error. We require the RMS misfit of the converged model to fall within 1.0 and 1.2. With this criterion we avoid overfitting the data. A priori constraints are imposed on each of the model parameters, thus

$$
\rho_{i}^{l} \leq \rho_{j} \leq \rho_{j}^{u}
$$

where $u$ means upper bound and $l$ lower bound. Any parameter may be made fixed by setting the upper and lower bounds to be the same, while any parameter can be made free by expanding the upper and lower bounds so they lie outside the range of physically realistic values. These constraints are very useful to introduce information from boreholes, to propose a model or to introduce the sea as in the Minamikayabe data.

\section{Synthetic Data Test}

The algorithm was checked against synthetic data from a chessboard model (Fig. 1(a)). This model has only 16 parameters, but it is very heterogeneous in terms of modeling, with high conductivity contrast between adjacent cells, and difficult to recover in terms of inversion. We computed the amplitudes and phases (TE and TM modes) of such a model at 8 sites located at $y=-3.5,-2.5,-1.5,-0.5,0.5,1.5$, 2.5 and $3.5 \mathrm{~km}$, at five frequencies $(10,3,1,0.3$ and 0.1 $\mathrm{Hz}$ ). This resulted in a set of 160 data. Five percent Gaussian noise was added to the data. For the inversion process, a population of 20 random models (10 pairs) was used. After an initial, stochastic set of model parameters was generated, the responses of the models were computed and compared with the noisy data by means of Eq. (2). After several hundred generations, the population of models had converged to a similar, low misfit form. The convergence criterion (3) can be used to halt the algorithm once acceptable misfit has been obtained.

The best model was obtained after 330 generations (Fig. 1(b)). Most of the prisms close to surface are very similar to the true model. Differences appear only in the deeper prisms. These differences arise because of conduc- 


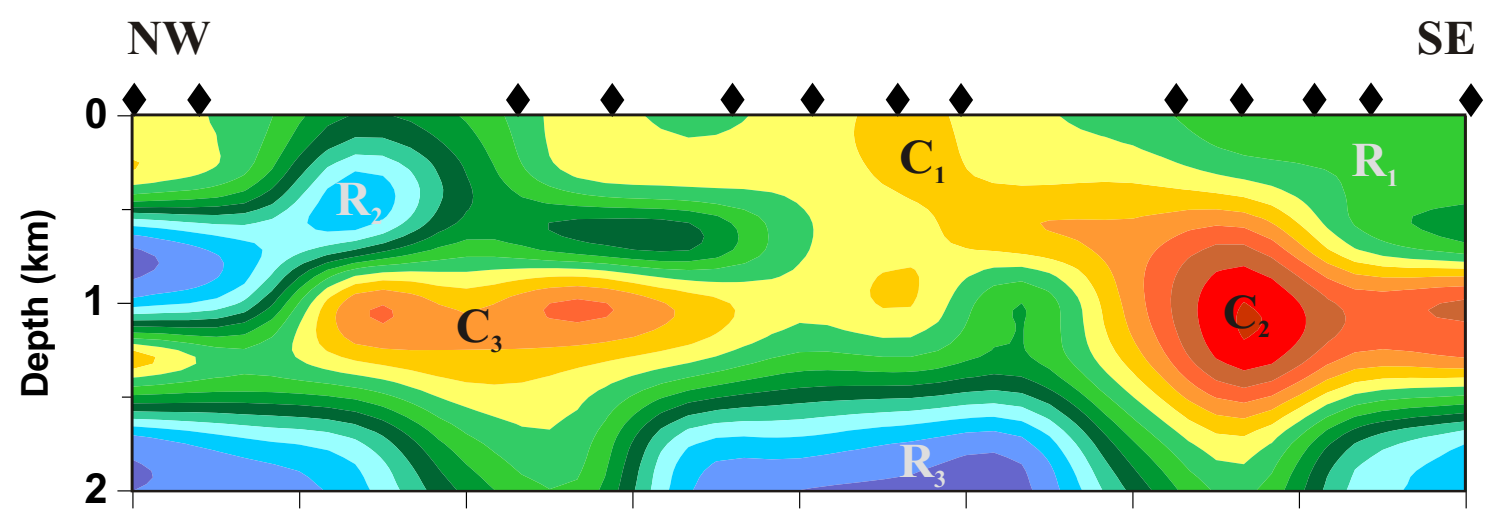

(a)

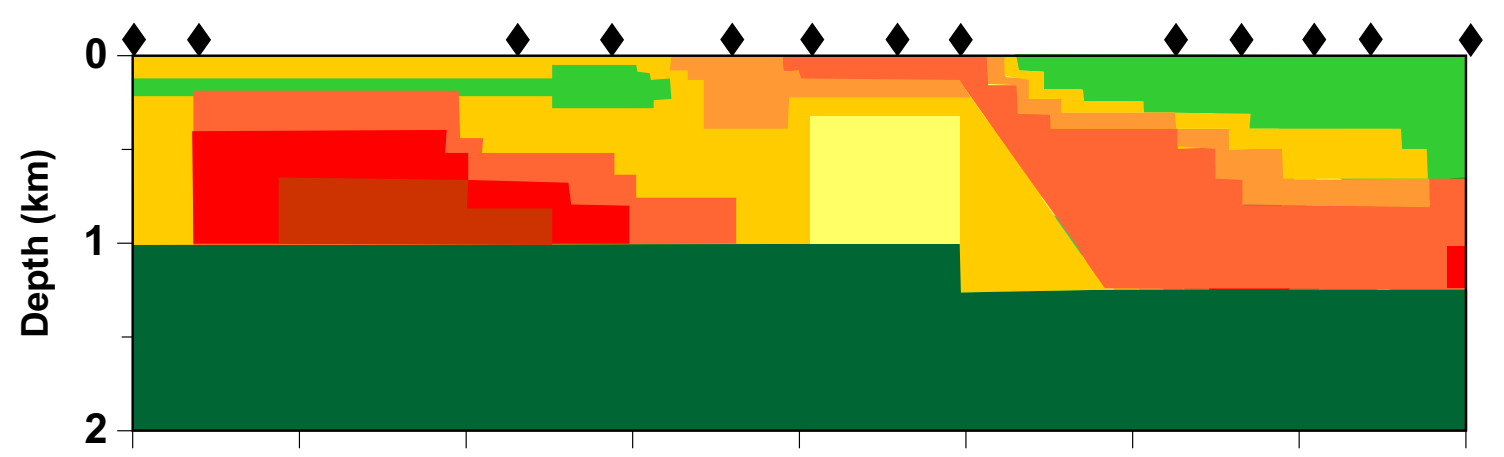

(b)

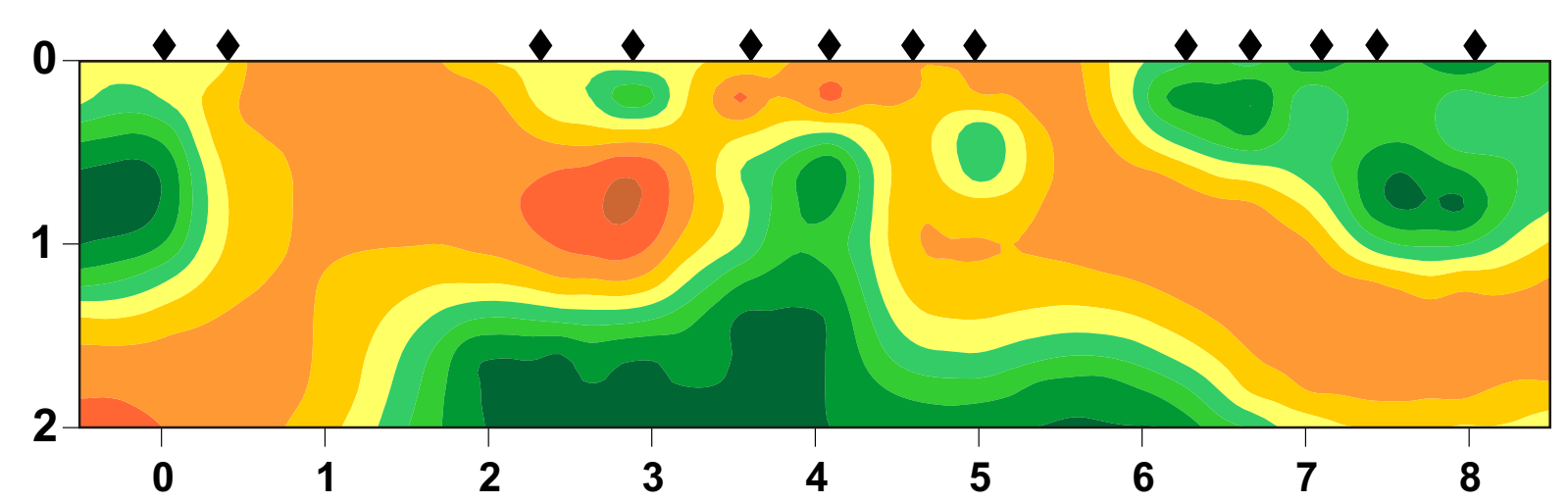

(c)

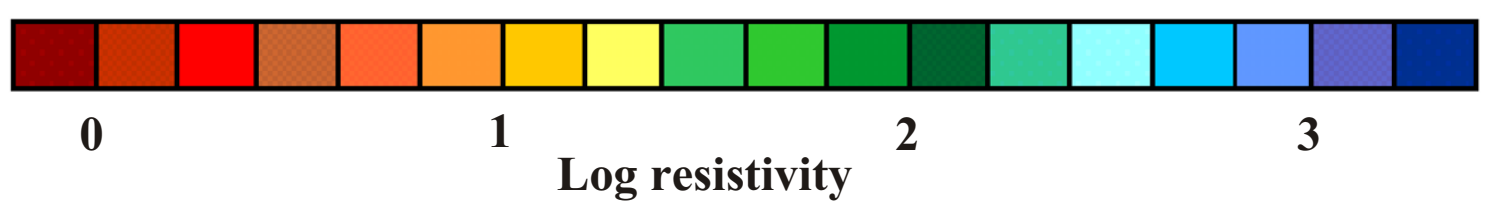

Fig. 4. (a) Model A obtained by the RGA. (b) Model B obtained by forward modeling. (c) Model C obtained by approximate imaging (Romo et al., 1997).

tance equivalence or conductivity screening in the deeper parts of the model, and by the noise added to the synthetic data.

Figure 2 shows the convergence properties of the best model. During the early generations, the model population is strongly heterogeneous and large changes and improvements in the model parameters occur over short periods of time. As the population matures, models are less heterogeneous and the improvement in the objective function for the best fitting models is more gradual. Toward the end of the iteration process, the population is very homogeneous because the best parameters are predominant in most of the new models generated. As the very mature population grows ever more homogeneous, additional genetic diversity is introduced through mutation. This will tend to assist in avoiding convergence to local minima. At the final stage, multiple mutations per model slow the convergence, so we stop crossover and allow only one mutation per model. 


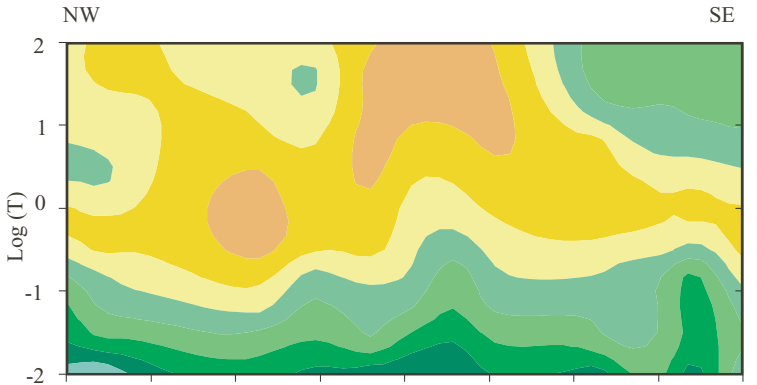

(a)

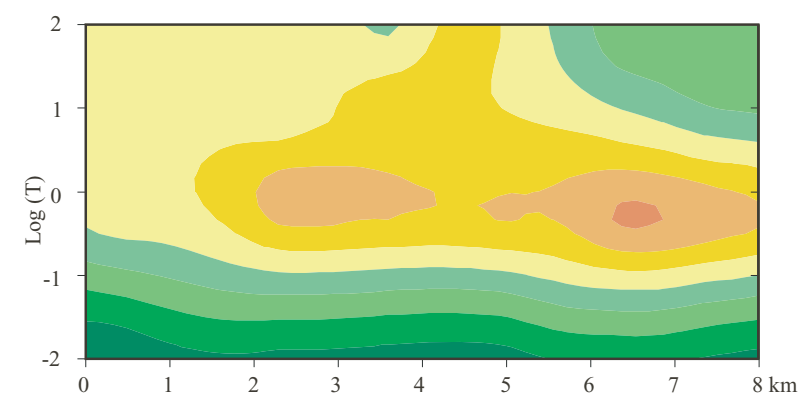

(c)

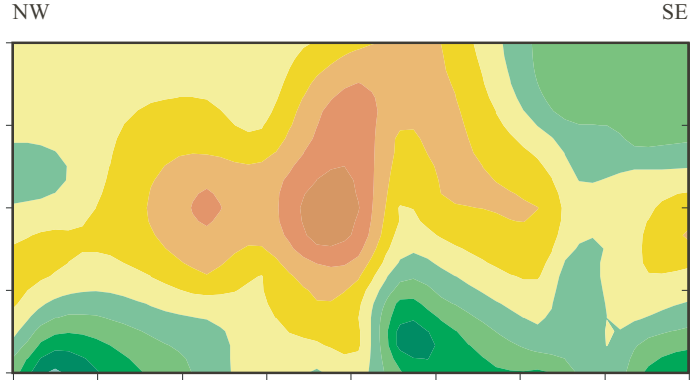

(b)

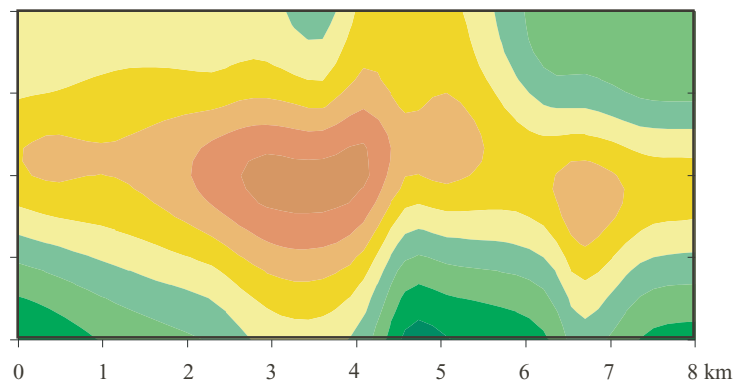

(d)

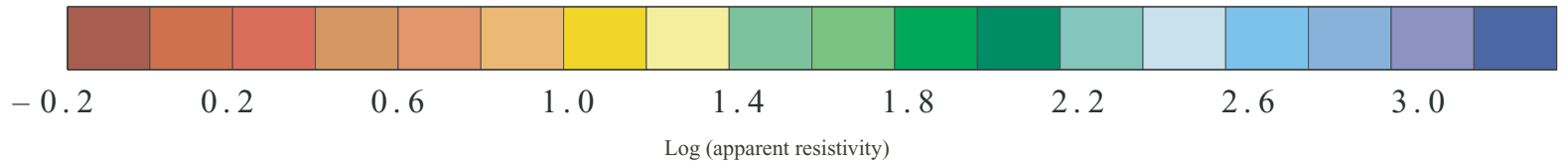

Fig. 5. Comparison between Ahuachapán data and the response of the best RGA model. (a) and (b) Data; apparent resistivity for TE and TM modes respectively. (c) and (d) Response; apparent resistivity for the TE and TM respectively.

\subsection{Ahuachapán, El Salvador}

Ahuachapán is an El Salvadorian geothermal area associated with volcanic activity related to the subduction of the Cocos Plate under the Caribbean Plate. A number of volcanic cones and hydrothermal surface manifestations exist in this area.

Geothermal reservoir exploitation began in the 1960s. More recently, an assortment of geophysical techniques were applied in order to improve the knowledge of the reservoir. In 1990 more than 120 MT soundings were collected and arranged in lines (Fig. 3). In this paper we interpreted only line N2, in order to demonstrate the usefulness of RGAs as a MT inversion technique in a real-world geothermal context.

Line N2 consists of 13 soundings located at $y=\{0,0.4$, $2.3,2.9,3.6,4.1,4.6,5.0,6.3,6.6,7.1,7.4$ and $8.0 \mathrm{~km}\}$. Every sounding consists of 11 frequencies, $f=\{100,39.8$, $15.84,6.31,2.51,1.0,0.398,0.158,0.063,0.025$ and 0.01 $\mathrm{Hz}$. For the inversion we assumed a 2-D model domain of 48 prisms arranged in 6 rows and 8 columns. Every prism is of $1 \mathrm{~km}$ of horizontal length, with a logarithmically increasing vertical length with depth. The model is bounded on the sides by a 1-D earth, and below by a homogeneous half-space. For the inversion we used a pool of 30 randomlygenerated models. Unfortunately, only apparent resistivities were available for both modes (TE and TM). Therefore, the phases were excluded from Eq. (3). After 570 generations we arrived at model A shown in Fig. 4(a). The conductor $\mathbf{C}_{3}$ corresponds to the reservoir currently being exploited

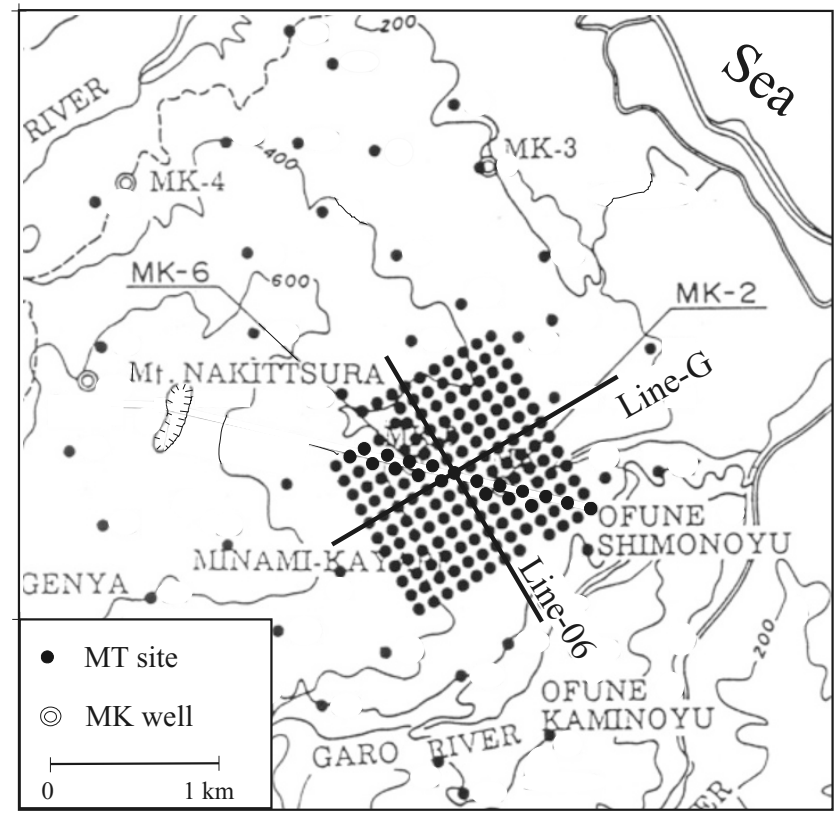

Fig. 6. Location of the MT soundings, line-G and line-06 in the Minamikayabe geothermal area, Japan.

for geothermal power. The surface conductor $\mathbf{C}_{1}$ matches the geothermal manifestation Cerro Blanco. The conductor $\mathbf{C}_{2}$ may be associated with the Hoyo de Coajuste volcano.

Our best-fitting model was compared with previously 

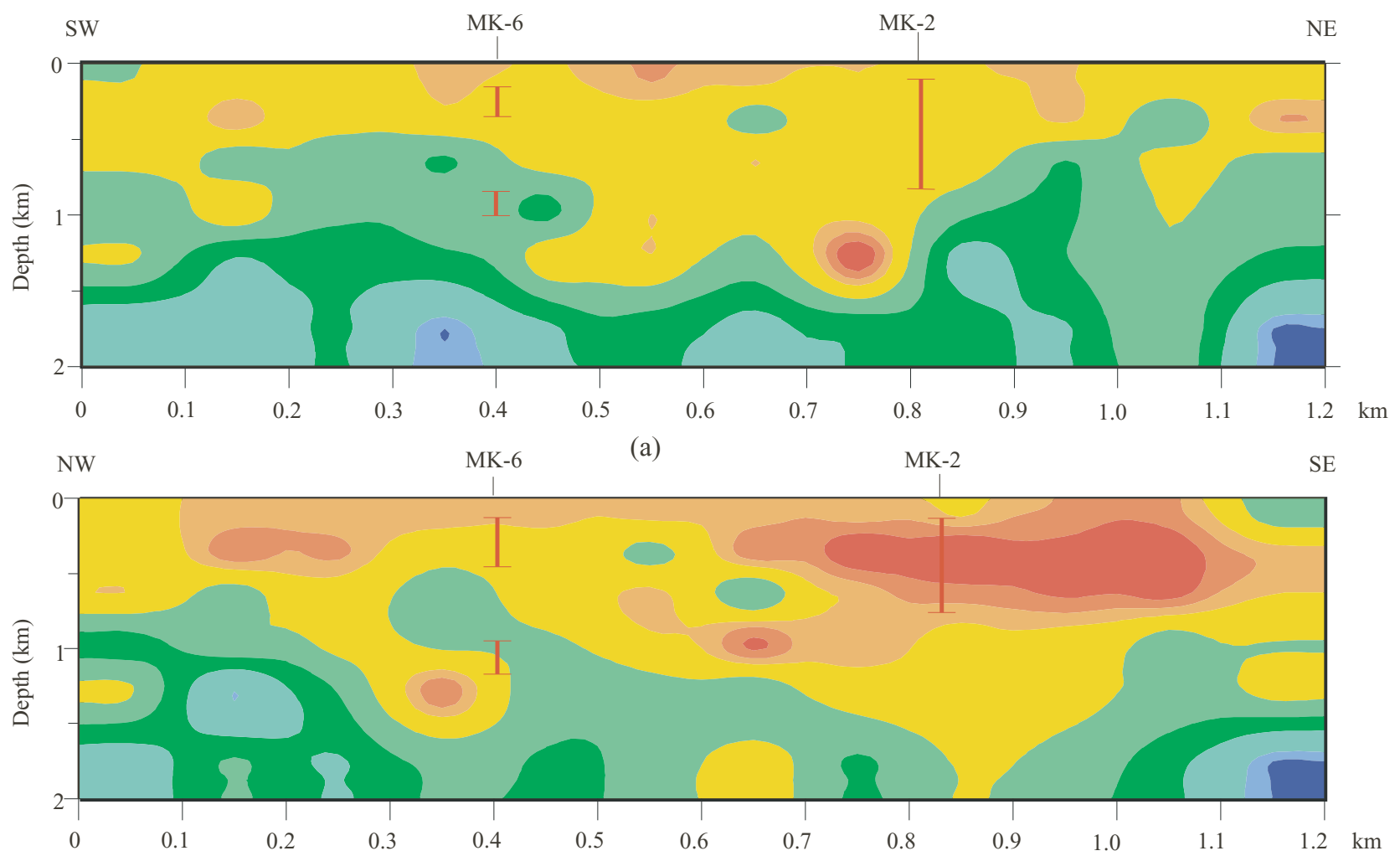

(b)

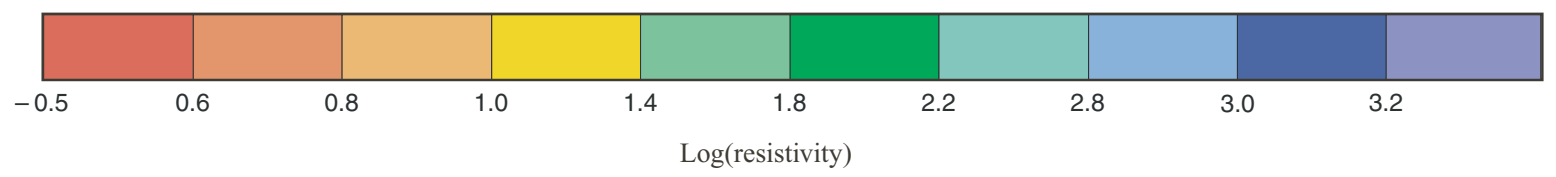

Fig. 7. (a) Model obtained for the MT line-G. In this line the presence of the sea was taken in consideration. There is a central conductor that correlates with the conductors detected along wells MK-6 and MK-2. (b) Model obtained for line-06. There is a conductor that thickens to the SE. The bar indicates the conductor detected along the wells.

published models. Figure 4(b) shows model B obtained by Romo et al. (1997), and in Fig. 4(c) model C is shown, as obtained by an approximate imaging technique valid only for low resistivity contrasts (Romo et al., 1997). Every technique has its own limitations, but GAs/RGAs have the advantage that they do not involve approximations that are associated commonly with other methods. Despite the very different approaches taken, the three independent interpretations of this data set reveal nearly the same view of the conductive features, as well as the resistive feature $\mathbf{R}_{1}$. The resistive feature $\mathbf{R}_{3}$ appears to correspond to the granitic basement, but in model B was aligned horizontally. There is a similar view of this feature in models $\mathrm{C}$ and $\mathrm{A}$. The resistive feature $\mathbf{R}_{2}$ is not present in models B and C, rather they extend the conductor $\mathbf{C}_{3}$ to the surface. Between $y=0.3$ and $y=2 \mathrm{~km}$ there are no soundings, therefore, the RGA is not able to constrain model $\mathrm{A}$ in that area. In Model $\mathrm{B}$ the interpreter extends conductor $\mathbf{C}_{3}$ on the basis of prior geological knowledge.

A comparison between the data and the responses from the best model obtained by the RGA is shown in Fig. 5. The lowest RMS misfit was 4, assuming a constant standard error of $3 \%$ in the data. As shown, the responses are similar to the data, although in strict statistic terms the model misfit remains high.

\subsection{Minamikayabe, Japan}

This interpretation was done as a contribution to the MTDIW3 project. The Minamikayabe geothermal area is located in southern Hokkaido Island. Under the program of "Geothermal Development Promotion Survey" several exploration wells were drilled and at the time this interpretation was done two wells were productive (MK-6 and MK2). In order to have a better understanding of the reservoir, a dense mesh of 209 MT soundings were established around the wells, with 161 located within a square mesh (Takasugi et at., 1992). We interpret here data from the two central lines; line-G and line-06 (Fig. 6). Each line has a length of $1200 \mathrm{~m}$ with 13 soundings every $100 \mathrm{~m}$. Soundings were recorded in the period range of 0.00005-1000 sec. Because of the very short length of the lines, we decided to interpolate the soundings to 13 periods; $\log T=\{-2.5,-2.25$, $-2.0,-1.75,-1.50,-1.25,-1.0,-0.75,-0.5,-0.25$, $0.0,0.25$ and 0.5$\}$, where the skin depths range from 100 to $2800 \mathrm{~m}$ in a homogeneous half-space of $24 \mathrm{ohm}-\mathrm{m}$ resistivity. Considering apparent resistivity and phase of both modes, there are 676 observations in every line.

In the first 150 generations of the RGA inversion, a mutation parameter was used that permitted multiple parameter 


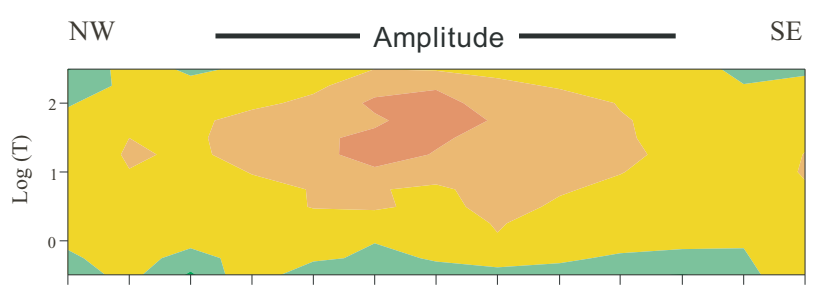

(a)

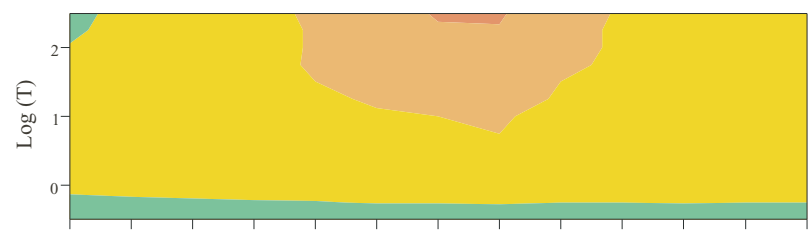

(c)

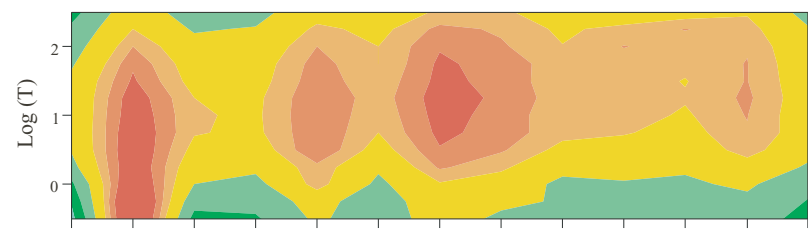

(e)

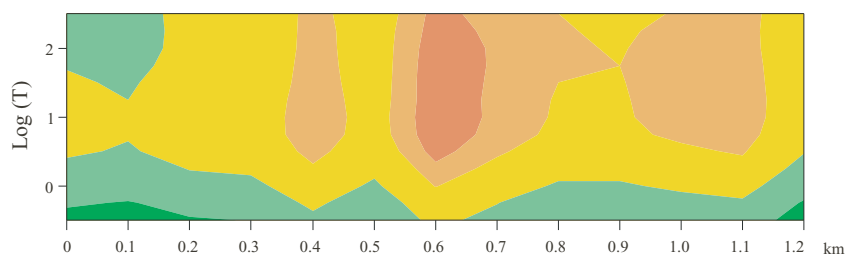

(g)

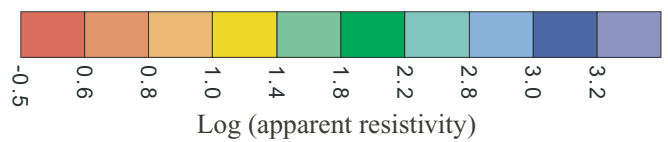

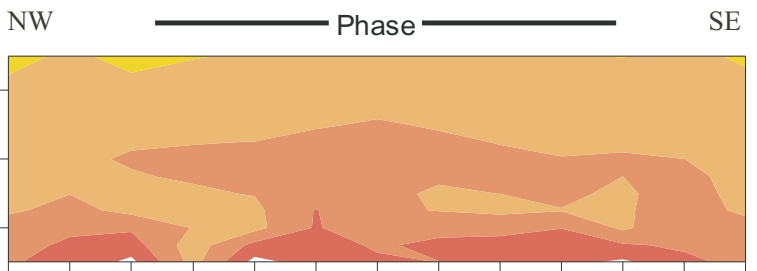

(b)

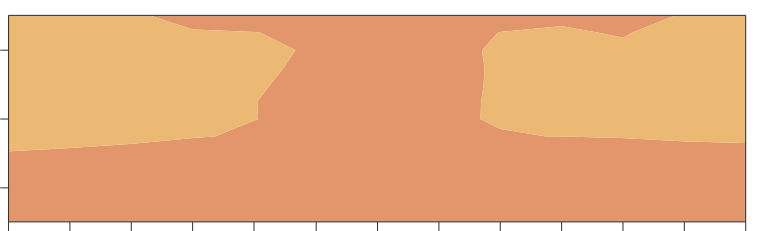

(d)

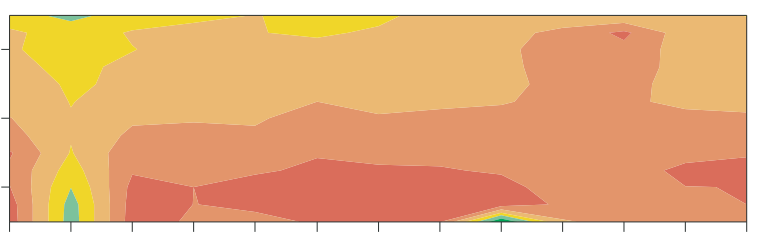

(f)

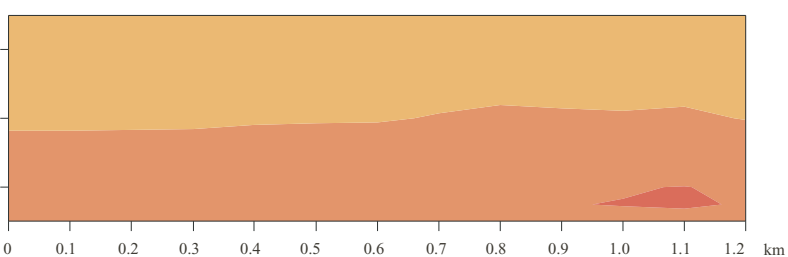

(h)

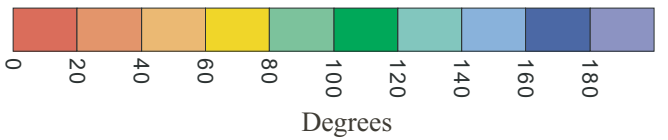

Fig. 8. Comparison between data and response for the best model in line-G. (a) and (b) Observed apparent resistivity and phase respectively for the TE mode. (c) and (d) Response for the TE mode. (e) and (f) Observed apparent resistivity and phase respectively for the TM mode. (g) and (h) Response for the TM mode.

changes per model. After the 150th generation, mutations were limited to only a single parameter change per model (see "Synthetic Data Test" section).

Along line-06, the subsurface was discretized in a grid of 72 prisms arranged in 12 columns and 6 rows, surrounded by a 1-D Earth at both sides and a homogeneous half-space below. Only 72 prism resistivities were considered as free parameters in the inversion, because the 1-D layers take the resistivity and thickness of the adjoining 2-D prisms, and the resistivity of the half-space is the average of the bottom prism resistivities.

A pool of 30 models was used in the RGA. The best model was obtained after 450 generations, achieving an RMS misfit of 2.6 (assuming a uniform 5\% standard error). Figure 7(b) shows the best fitting model. A large conductor is evident that thickens toward the south east. This conductor may correspond with the Siodomarigawa formation, a fractured, intrusive rock (Takasugi et al., 1992). The resistive zone below may correspond to non-fractured basaltic rock. Figure 7(b) shows the location of conductors reported by down-hole resistivity logs taken within the geothermal wells. These conductors correspond to those in the RGA inverse model.

For line-G the subsurface was discretized in 72 prisms, a 1-D earth at both sides and a half-space below. The sea and coastline to the south east of the line was introduced to the inversion by means of equality constraints on the model (Eq. (4)). Considering a pool of 30 models, the best model was obtained after 520 generations, with an RMS misfit of 2. This model (Fig. 7(a)) shows a thick conductor in the center of the resistivity section. The down-hole conductors reported by Takasugi et al. (1992) are also shown in the figure.

In Fig. 8 the data and responses from the best model for line-G are seen. TM-mode was fit much better than TEmode, but in general phases and amplitudes were properly reproduced.

Cerv and Pek (1997) reported other models for the same 
lines by using a random searching technique.

\section{Conclusions}

The accuracy of the forward solution is sufficiently high that the heterogeneous high-contrast models generated randomly by the Recombinant Genetic Analogue proves no impediment to the overall convergence of the inversion process. This is attributed to the automatic grid generator, which depends on both the frequency and conductivity distribution within the model.

The exercise with noisy synthetic data demonstrates that it is possible to use the RGA as an inversion method for 2-D magnetotellurics, even when the model is highly heterogeneous and the data are contaminated with noise. The synthetic example revealed that, as with other inversion techniques, the problems of resolution and conductance equivalence are intrinsic to MT.

The results from El Salvador show that the nonlinear RGA inversion has obtained almost the same model features as other, earlier techniques. The model revealed conductors that appear to be associated directly with the geothermal field, with surface geysers, and with Hoyo de Coajuste volcano. The way in which prior bounds may be put on any given model parameter makes the RGA easily adapted to 2-D MT hypothesis testing. For example, one could test whether small conductive features that have been revealed in an RGA or other inversion are strictly required by the data. This is accomplished by imposing an a priori high resistance bound on the resistivity of the prisms associated with that feature, and then by inverting the same data set a second time. If the data can still be fit with that feature suppressed, then the feature is not strictly required by the data. Hypothesis testing is one of the most compelling advantages of this approach.

For the Kayabe data we show that, as for the case of hypothesis testing, it is possible to introduce geological information to the solution by means of a priori constraints. For case of line-G the presence of the sea was taking into consideration. For the models corresponding to the MT central lines, a conductor located at the center matches information reported by resistivity logs obtained inside the exploratory wells.

RGAs can be added to the pantheon of practical (although computationally demanding) 2-D MT inversion techniques, particularly when prior bounds and hypothesis testing are key elements of the question under investigation.

Acknowledgments. The authors acknowledge the British Council, CONACYT and CICESE for the economical support during this project. We thank Dr. Takasugi and NEDO for allowing us to use the Minamikayabe MT data set, and CEL for the use of the Ahuachapán data. We also thank the editors for their indulgence in an 11th hour submission. This work was carried out when the AS was a member of the Institute of Theoretical Geophysics, Cambridge, and MPF was a British Council Scholar attached to the ITG.

\section{References}

Brewitt-Taylor, C. R. and J. Weaver, On the finite difference solution of two-dimensional induction problems, Geophys. J. R. Astr. Soc., 47, 375396, 1976.

Cerv, V. and J. Pek, 2-D interpretation of Minamikayabe MT data set by the controlled random search technique, J. Geomag. Geoelectr., 49, 17171729, 1997.

Everett, M. E. and A. Schultz, Two-dimensional nonlinear magnetotelluric inversion using a Genetic Algorithm, J. Geomag. Geoelectr., 45, 10131026, 1993.

Poll, H. E., Automatic Forward Modeling of two-dimensional problems in electromagnetic induction, Ph.D. Thesis, University of Victoria, 1994.

Romo, J. M., C. Flores, R. Vega, R. Vazquéz, M. A. Pérez, E. Gómez, F. Esparza, J. Quijano, and V. García, A closely-spaced magnetotelluric study of the Ahuachapán-Chipilapa geothermal field, El Salvador, Geothermics, 26, 627-656, 1997.

Sambridge, M., Finding acceptable models in nonlinear inverse problems using a neighbourhood algorithm, Inverse Problems, 17, 387-403, 2001.

Takasugi, S., K. Tanaka, N. Kawakami, and S. Muramatsu, High spatial resolution of the resistivity structure revealed by a dense network MT measurements. A case study in the Minamikayabe area, Hokkaido, Japan, J. Geomag. Geoelectr., 44, 289-308, 1992.

Weaver, J. and C. R. Brewitt-Taylor, Improved boundary conditions for the numerical solution of E-polarization problems in geomagnetic induction, Geophys. J. R. Astr. Soc., 54, 309-317, 1978.

Weaver, J. T., Mathematical Methods for Geo-electromagnetic Induction, Research Studies Press, 1994.

M. A. Pérez-Flores (e-mail: mperez@cicese.mx) and A. Schultz (e-mail: adam@ocean.cf.ac.uk) 